Proc. Indian Acad. Sci. (Earth. Planet. Sci.), Vol. 89, Number 2, July 1980, pp. 231-238. (C) Printed in India.

\title{
Capability of Bhaskara SAMIR to distinguish atmospheric water vapour and liquid water contents
}

\author{
P C PANDEY, A K SHARMA and B S GOHIL \\ Meteorology Division, Remote Sensing Area, Space Applications Centre, \\ Ahmedabad 380 053, India \\ MS received 11 January 1980; revised 25 April 1980
}

\begin{abstract}
An environmental model to compute microwave brightness temperatures for downward looking radiometers on board satellite is described. The effects of water vapour, oxygen and clouds on the brightness temperature have been studied for frequencies from 5 to $50 \mathrm{GHz}$ for a standard tropical atmosphere. The effect of look angle on brightness temperature has also been investigated. Based on the model it has been shown that while the radiometers on board Bhaskara at 19.35 and $22.235 \mathrm{GHz}$ are capable of giving the atmospheric water vapour and liquid water contents, the ability to distinguish these quantities is more for the combination of the frequencies $22 \cdot 235$ and $31 \mathrm{GHz}$.
\end{abstract}

Keywords. Water vapour; liquid water; brightness temperature; Bhaskara SAMIR ; microwave radiometer.

\section{Introduction}

Atmospheric water vapour and liquid water contents are important meteorological parameters. Monitoring these quantities over ocean by conventional techniques is difficult. Remote sensing from satellites with passive microwave radiometers offers a good possibility for getting this information.

The Indian Satellite Bhaskara has on board passive microwave radiometers operating in the frequencies $19 \cdot 35$ and $22.235 \mathrm{GHz}$. Preliminary attempts have been made (Pandey et al 1979) to derive water vapour and liquid water contents in the atmosphere with Bhaskara data for selected passes over Bay of Bengal and Arabian Sea.

The present paper deals with a detailed analysis of the factors involved in the distinction of water vapour and liquid water contents in the atmosphere by microwave radiometry in the frequency range 5 to $50 \mathrm{GHz}$. A suitable environmental model for carrying out the studies has been described. The results are discussed with special reference to the Bhaskara frequencies.

p. (A) -12 


\section{Radiation received by a satellite microwave radiometer}

The microwave radiation reaching a satellite radiometer can be expressed in terms of the following :

(i) The radiation emitted by the sea.

(ii) The upwelling radiation emitted by the atmosphere.

(iii) The down-welling radiation emitted by the atmosphere and extra terrestrial sources and reflected upward from sea surfaces.

(iv) The attenuation of the radiation emitted and reflected from the sea surface by the intervening atmosphere.

For convenience of computations, the atmosphere has been divided into layers each $1 \mathrm{~km}$ thick and is characterised by pressure, temperature and humidity. A standard tropical atmosphere with exponential variation of pressure and humidity has been used in the computation. The model is sensitive to the altitude distribution of atmospheric water vapour and this is automatically taken into account while defining the dimensionless quantity ' ability factor' for relative comparison of a pair of frequencies. The top of the atmosphere has been set at $30 \mathrm{~km}$ above which the water vapour is assumed to be negligible.

\section{The environmental model}

The emissivity of the sea water plays a key role in determining the radiation emitted by the surface. The emissivity has been calculated by assuming a smooth air-water interface using Fresnel relations to calculate reflectivity from dielectric properties of the surface as a function of viewing angle and polarisation. The emissivity is then given by

$$
\epsilon=1-|R(\theta, \phi)|^{2} \text {. }
$$

The polynomial fits by Holinger (1973) for static dielectric constant, relaxation time and ionic conductivity in terms of salinity and temperature have been used in the computation. These polynomials are based on the data of Saxton and Lane (1952). Since no laboratory measurements of dielectric properties of sea water were available, the measurement on aqueous sodium chloride was approximated to be sea water as far as its electrical properties are concerned.

The above regression equations were used to predict $\epsilon^{\prime}$ and $\epsilon^{\prime \prime}$ for any given temperature, salinity and frequency. These quantities were then used to compute emissivity using Fresnel's formula (Pandey and Sharma 1980). The dielectric properties of pure water were obtained from the above general expressions by setting salinity to zero.

The upwelling and downwelling radiation is obtained from the solution of radiative transfer equation. The intensity of radiation is described in terms of brightness temperature under Rayleigh-Jeans approximation.

The model used for computing absorption coefficients due to water vapour is the one given by Staelin (1966) based on the interpretation of Barret and Chung's (1962) formula. 
The model for computing absorption coefficients due to oxygen molecule is the one given by Rosenkranz (1975). The details of the computation and results for a standard tropical atmosphere are described by Pandey et al (1979).

The model for calculating absorption coefficients due to cloud is the one suggested by Paris (1971) and extended by Pandey et al (1979) using the polynomial fits of Hollinger and co-workers (Hollinger 1973; Hollinger et al 1975) in terms of real and imaginary parts of relative dielectric constants and liquid water contents.

\section{Computational procedure}

For a non-scattering atmosphere in local thermodynamic equilibrium, the zenith brightness temperature is given by the solution of radiative transfer equation (Chandrasekhar 1960) as

$$
T_{B}=\int_{0}^{\infty}\left[T(z) \alpha(z) \exp -\int_{0}^{Z} \alpha\left(z^{\prime}\right) d Z^{\prime}\right] d Z
$$

where $T(z)$ is the atmospheric temperature at height $z$ and $a(z)$ is the total absorption at height $z$.

The apparent temperature ideally measured by a radiometer at an altitude $h$ above the surface of the ocean is given by

$$
\begin{aligned}
T_{B}=\mathrm{r} & {\left[T_{c} \exp \left[-\sec \theta \int_{0}^{\infty} a(z) d z\right]+\int_{0}^{\infty} T(z) \alpha(z) \sec \theta\right.} \\
& \times \exp \left[-\sec \theta \int_{0}^{z} a(z) d Z\right] \exp \left[-\sec \theta \int_{0}^{h} a(z) d z\right] \\
& +\epsilon T_{S} \exp \left[-\sec \theta \int_{0}^{h} a(z) d z\right]+\int_{0}^{h} T(z) a(z) \sec \theta \\
& \times \exp \left[-\int_{Z}^{h} a\left(z^{\prime}\right) d z^{\prime}\right] d Z .
\end{aligned}
$$

The first term of equation (3) is the cosmic background radiation whose contribution is small and has not been included in the computation. The second term is called sky brightness temperature and for computer implementation, it can be written as

$$
\begin{aligned}
& T_{\text {sky }}=\sum_{i=1}^{n-1}\left[\left(1-t_{i}\right) \bar{T}_{i} \prod_{j=0}^{i-1} t_{i}\right] \\
& t=\text { transmittance }=\exp \left[-\sec \theta \int a(z) d z\right] .
\end{aligned}
$$

This is the downwelling radiation received at the surface. The term with emissivity in equation (3) is the surface contribution to the brightness temperature. 
The last term of equation (3) is upward propagating emission from the intervening atmosphere between ocean surface and satellite height. For computational purposes,

$$
T_{\text {atm }}=\sum_{i=1}^{n-1}\left[\left(1-t_{i}\right) \bar{T}_{i} \prod_{j=i+1}^{n} t_{j}\right]
$$

Thus equation (3) is written as

$$
T_{B}=\left[T_{\text {fkg }}\left(1-\epsilon_{\mathrm{S}}\right)+T_{S} \epsilon_{\mathrm{S}}\right] t+T_{\mathrm{atm}} .
$$

Equations (4), (5) and (6) are the main equations from which computational results are presented.

\section{Results and discussions}

The variation of brightness temperatures generated from the model described in $\S 3$ for frequencies from 5 to $50 \mathrm{GHz}$ is shown in figure 1 for a standard tropical atmosphere. The first curve is for a clear atmosphere with molecular oxygen and water vapour as the only absorbers. The increased brightness temperature at water vapour resonance is evident from the figure. The curve shows a minima around $30 \mathrm{GHz}$ after which it increases continuously suggesting the influence of

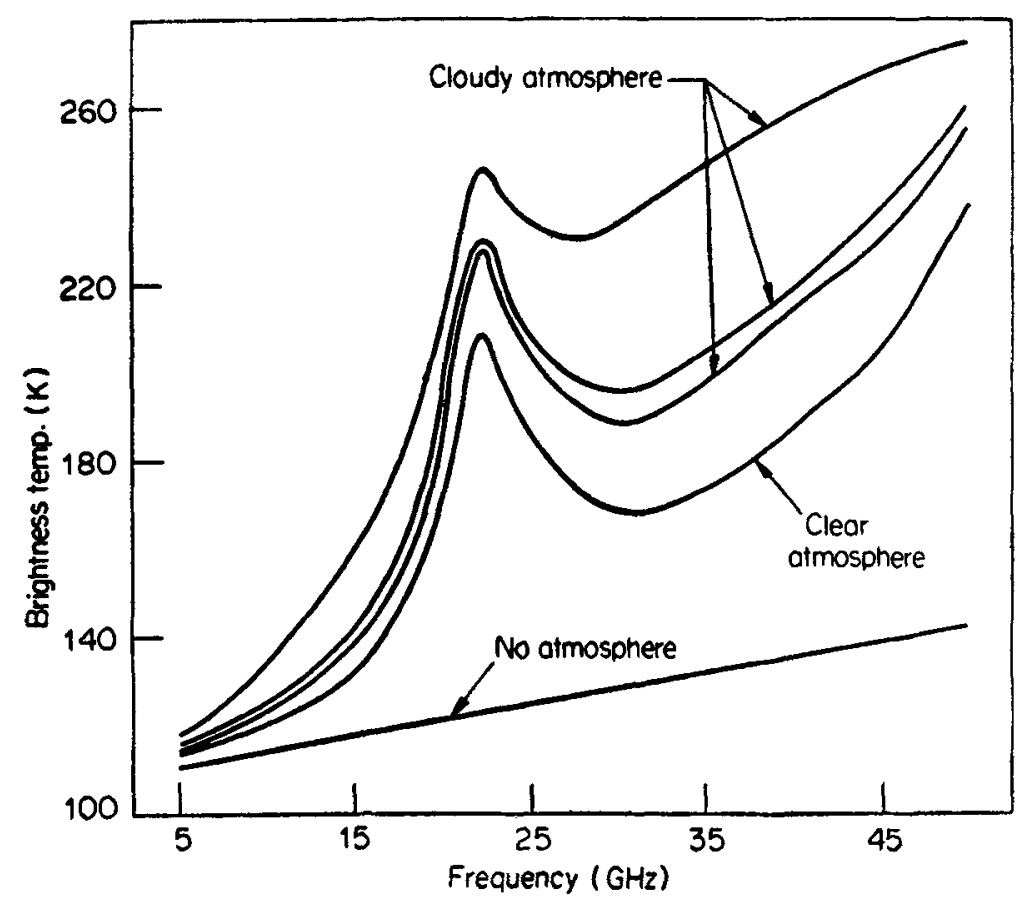

Figure 1. The effect of clouds on brightness temperature. 
$5 \mathrm{~mm}$ oxygen band. Curves II, III and IV are obtained after introducing clouds with different amounts of liquid water contents (table 1). It is found from curve I and II that a cloud with a liquid water content of $0.04 \mathrm{~g} / \mathrm{cm}^{2}$ produces a change of only about $8^{\circ} \mathrm{K}$ in brightness temperature near $19 \mathrm{GHz}$ whereas at frequencies near $31 \mathrm{GHz}$, a change of about $21^{\circ} \mathrm{K}$ is observed.

The line in figure 1 with no atmosphere represents the surface contribution to the total brightness temperature which is equal to the product of emissivity and sea surface temperature. While calculating the emissivity the sea surface salinity is taken to be $35 \mathrm{ppt}$ and sea surface temperature to be $30^{\circ} \mathrm{C}$. The increase in brightness temperature is due to frequency dependence of the emissivity. The contribution of the atmosphere to the total brightness temperature is about $80^{\circ} \mathrm{K}$ at $22.235 \mathrm{GHz}$ and about $34^{\circ} \mathrm{K}$ and $48^{\circ} \mathrm{K}$ for $19 \mathrm{GHz}$ and $31 \mathrm{GHz}$ frequencies.

It is also seen from figure 1 that the effect of atmosphere becomes significant only for frequencies greater than about $10 \mathrm{GHz}$. For frequencies below about $30 \mathrm{GHz}$, the major contribution to the total brightness temperature comes from water vapour. The role of oxygen becomes significant for higher frequencies i.e. near $60 \mathrm{GHz}$ oxygen complex.

Figure 2 shows the variation of brightness temperature with different look angles from 0 to $70^{\circ}$. For frequencies below $15 \mathrm{GHz}$, the brightness temperature corresponding to the horizontal polarisation $\left(T_{B H}\right)$ decreases with look angle whereas the brightness temperature corresponding to the vertical polarisation $\left(T_{B V}\right)$ increases monotonically with look angles. For frequencies above around $30 \mathrm{GHz}$, the $T_{B H}$ initially remains constant upto around $20^{\circ}$ look angle and then slowly decreases with minimum around $50^{\circ}$ look angle and then increases further. It can also be seen that for a particular look angle near $50^{\circ}$, change in $T_{B H}$ from 20 to $25 \mathrm{GHz}$ is more $\left(21^{\circ}\right)$ than $T_{B V}$ which is about $11^{\circ} \mathrm{K}$. For a particular frequency (say $20 \mathrm{GHz}) T_{B V}$ is more sensitive $\left(27^{\circ} \mathrm{K}\right)$ than $T_{B H}\left(8^{\circ} \mathrm{K}\right)$ for a change of $10^{\circ}$ look angle from $40^{\circ}$ to $50^{\circ}$. The contribution of atmosphere to the total brightness temperature at different look angles shows the pronounced effect for angles greater than about $30^{\circ}$.

Table 1. Model of the clouds used in computation.

\begin{tabular}{|c|c|c|c|c|}
\hline \multirow{2}{*}{$\begin{array}{c}\text { Height } \\
\text { (km) }\end{array}$} & \multicolumn{4}{|c|}{ Liquid water density in $\mathbf{g} / \mathbf{m}^{3}$ for } \\
\hline & I & $\Pi$ & III & IV \\
\hline $0 \cdot 0$ & No cloud & 0 & $\mathbf{0}$ & $\mathbf{0}$ \\
\hline $1 \cdot 0$ & & $\mathbf{0}$ & $\mathbf{0}$ & $\mathbf{0}$ \\
\hline $2 \cdot 0$ & & 0.0051 & 0.05 & $0 \cdot 21$ \\
\hline $\mathbf{3} \cdot \mathbf{0}$ & & $0 \cdot 15$ & $0 \cdot 10$ & 0.63 \\
\hline $4 \cdot 0$ & & $0 \cdot 2409$ & $0 \cdot 40$ & $1 \cdot 05$ \\
\hline $5 \cdot 0$ & & 0.0039 & 0.05 & 0.40 \\
\hline $6 \cdot 0$ & & $\mathbf{0}$ & $\mathbf{0}$ & $\mathbf{0}$ \\
\hline
\end{tabular}




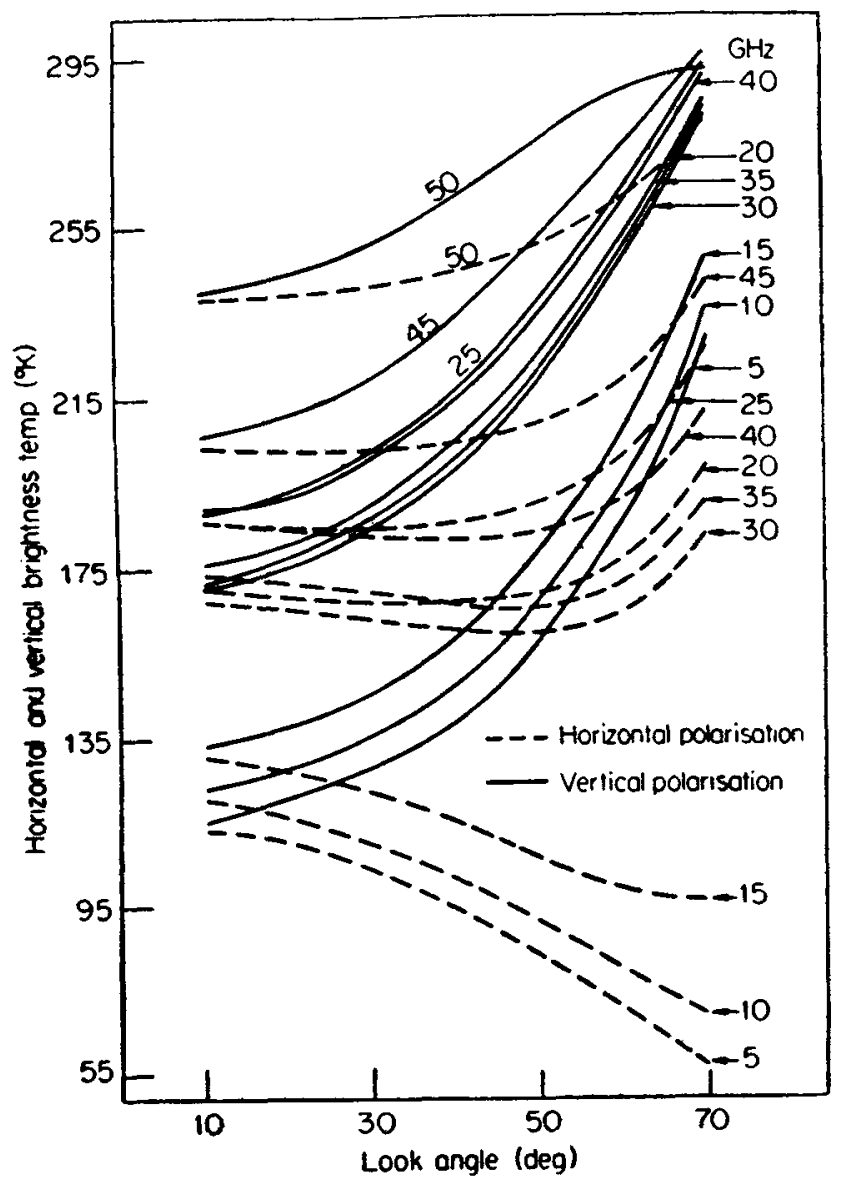

Figure 2. Variation of microwave brightness temperature with look angle at different frequencies.

\section{Distinction of water vapour and liquid water content}

Water vapour has a weak resonance line at $22.235 \mathrm{GHz}$ and therefore can be detected at that frequency. But the detection may be confused due to the presence of liquid water in the clouds. Therefore a second radiometer at some other frequency is required which may resolve this ambiguity.

In order to decide the choice of the second observing frequency, the environmental model was used to generate an ability factor from $5 \mathrm{GHz}$ to $50 \mathrm{GHz}$ frequencies that are generally used for satellite microwave radiometry. The ability factor provides a measure of the ability of a two frequency measurement to separate the effects of liquid water and water vapour.

The ability factor as defined by Hollinger (1979) is illustrated in figure 3. For different water vapours and liquid water contents in the atmosphere, the brightness temperatures for different frequencies $(5$ to $50 \mathrm{GHz}$ ) are plotted as ordinates versus the brightness temperature at $22.235 \mathrm{GHz}$ as abscissa. From this graph 


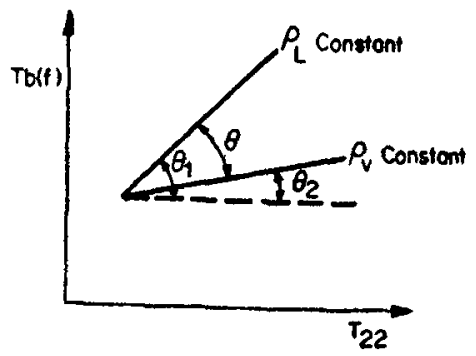

Figure 3. Definition of ability factor.

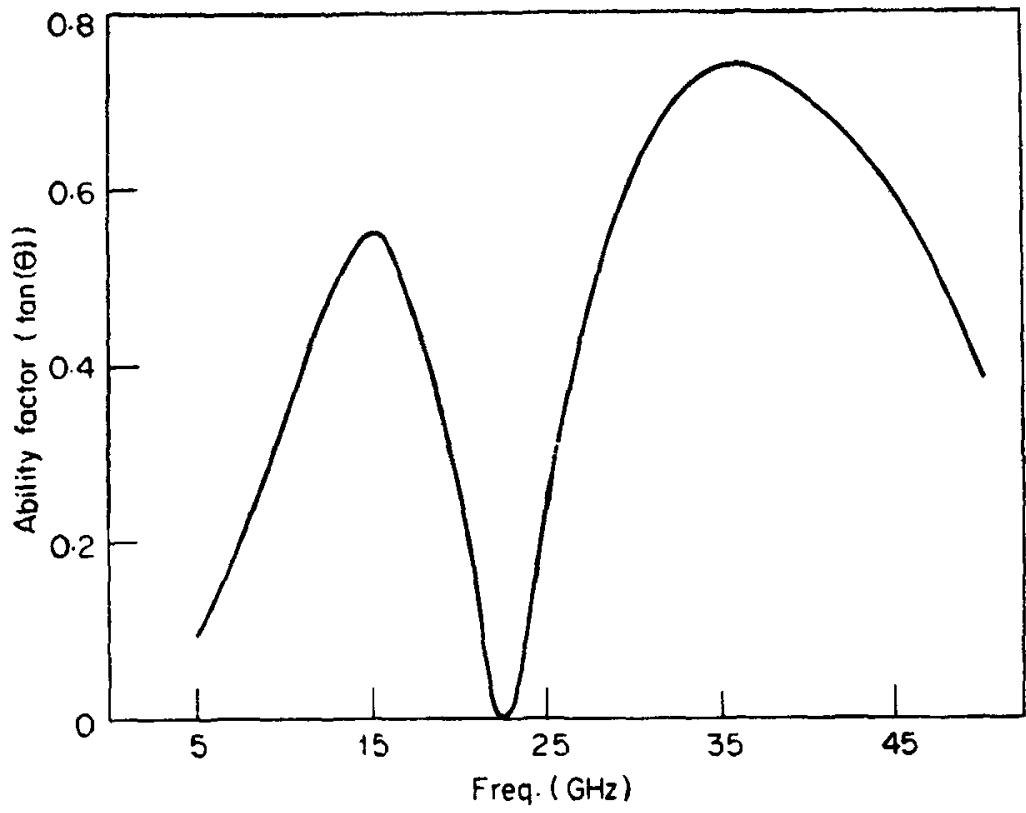

Figure 4. Ability factor versus frequencies for a dual frequency radiometer, one at $22.235 \mathrm{GHz}$ and the other between 5 and $50 \mathrm{GHz}$.

$$
\tan \theta_{1}=\left[\frac{\partial T_{B}(f)}{\partial \rho_{v}} / \frac{\partial T_{B}(22)}{\partial \rho_{v}}\right] \rho_{L}=\text { constt, }
$$

and $\tan \theta_{2}=\left[\frac{\partial T_{B}(f)}{\partial \rho_{L}} / \frac{\partial T_{B}(22)}{\partial \rho_{L}}\right] \rho_{v}=$ constt.

where $\rho_{L}=$ density of liquid water; $\rho_{v}=$ density of water vapour; $T_{B}(f)=$ brightness temperature at frequency $(5-50 \mathrm{GHz}$ in the present study) and $T_{B}(22)=$ brightness temperature at water vapour resonance, $22 \cdot 235 \mathrm{GHz}$.

From this, the ability factor is defined as

$$
\operatorname{Tan}(\theta)=\tan \left(\theta_{1}-\theta_{2}\right)=\frac{\tan \theta_{1}-\tan \theta_{2}}{1+\tan \theta_{1} \tan \theta_{2}}
$$

Figure 4 shows the variation of ability factor with frequencies from 5 to $50 \mathrm{GHz}$. It can be seen that the ability factor is maximum around $15 \mathrm{GHz}$ and $37 \mathrm{GHz}$. 
Although maximum ability is around the above two frequencies, the frequencies 19 and $31 \mathrm{GHz}$ are generally used probably due to hardware reasons. The ability of 22 and $31 \mathrm{GHz}$ radiometer is about $67 \%$ as compared to the ability of $32 \%$ for 19 and $22 \mathrm{GHz}$ in distinguishing water vapours and liquid water contents in the atmosphere. The error analysis was also performed to estimate the total atmospheric water vapour and liquid water content using 19-22 $\mathrm{GHz}$ frequencies and 22-31 GHz frequencies. The same set of atmospheric conditions were used to obtain regression equations in terms of brightness temperatures. Taking a measurement error of $1^{\circ} \mathrm{K}$ in brightness temperature at three frequencies, it was found that the uncertainty in $W$ and $Q$ with $19-22 \mathrm{GHz}$ frequencies are $0 \cdot 1730$ and $0 \cdot 1280$ respectively whereas for frequencies $22-31 \mathrm{GHz}$, it becomes $0 \cdot 1251$ and $0 \cdot 0530$. These results are in agreement with results obtained by Grody (1976).

\section{Acknowledgements}

The authors are grateful to Dr T A Hariharan, Head, Meteorology Division, for his keen interest in the present investigation and useful guidance and suggestions. Useful suggestions through correspondence from Dr James P Hollinger, Naval Research Laboratory, Washington DC, USA, is thankfully acknowledged. Thanks are also due to Dr V K Garde, Meteorology Division, for useful comments.

\section{References}

Barret A H and Chung V K 1962 J. Geophys. Res. 674259

Chandrasekhar S 1960 Radiative transfer (New York: Dover)

Grody Norman C 1976 IEEE Trans. Ant. Prop. AP 24155

Hollinger James P 1973 Naval Research Laboratory, Washington DC, Research Report No. 71102

Hollinger James P, Lerner R M and Wisler M M 1975 Naval Research Laboratory, Memorandum Report No. 3159

Hollinger James P 1979 Private Communication

Paris Jack F 1971 Transfer of thermal microwave in the atmosphere, Department of Meteorology, University of Texas, Austin, Vol. I and II.

Pandey P C, Gohil B S and Sharma A K 1979 Mausam (Communicated)

Pandey P C and Sharma A K 1980 Mausam 31201

Rosenkratz P W 1975 IEEE Trans. Ant. Prop. AP-23, 496

Saxton J A and Lane J A 1952 Wireless Engineer 29269

Staelin D H 1966 J. Geophys. Res. 712875 